\section{Fears and fallacies: Doctors' perceptions of the barriers to medical innovation}

\author{
Tracey Elliott' ${ }^{1}$, Jose Miola', Ash Samanta ${ }^{2}$ and Jo Samanta ${ }^{3}$
}

Clinical Ethics 2019, Vol. 14(4) 155-164 (C) The Author(s) 2019 (c) (i)

Article reuse guidelines: sagepub.com/journals-permissions DOI: 10.1 1 177//477750919886090 journals.sagepub.com/home/cet

(9SAGE

\begin{abstract}
In 2014, Lord Saatchi launched his ultimately unsuccessful Medical Innovation Bill in the UK. Its laudable aim was to free doctors from the shackles that prevented them from providing responsible innovative treatment. Lord Saatchi's principal contention was that current law was the unsurmountable barrier that prevented clinicians from delivering innovative treatments to cancer patients when conventional options had failed. This was because doctors feared that they might be sued or tried and convicted of gross negligence manslaughter if they deviated from standard practice. Concerns about fear of the law and potential negative effects on medical practice are not new. Fear of litigation has been suggested as the reason for doctors practising "defensive medicine," by opting for treatments regarded as "grievance-resistant," rather than clinically indicated, for example, by ordering diagnostic tests or performing certain procedures, which are not strictly medically necessary. Whilst this claim is plausible and apparently accepted by the courts, there is limited empirical evidence in support of it so far as practitioners in the UK are concerned. In this paper, we report on our empirical research which provides a snapshot of medical opinion to begin to rectify this gap. We ran focus groups of different medical specialties, asking what these medical practitioners thought the barriers to medical innovation to be. We found that fear of the law was not the principal barrier to be lowered, and that the answer was far more multifaceted.
\end{abstract}

\title{
Keywords
}

Medical innovation, barriers to innovation, empirical ethics

\section{Introduction}

In 2014, Lord Saatchi launched his ultimately unsuccessful Medical Innovation Bill in the UK. Its laudable aim was to free doctors from the shackles that he perceived prevented them from providing responsible innovative treatment. Lord Saatchi's principal contention was that current law was the unsurmountable barrier that prevented clinicians from delivering innovative treatments to cancer patients when conventional options had failed. This was because doctors feared that they might be sued or tried and convicted of gross negligence manslaughter if they deviated from standard practice. ${ }^{1,2}$ Concerns about fear of the law and potential negative effects on medical practice are not new. Fear of litigation has been suggested as the reason for doctors practising "defensive medicine," by opting for treatments regarded as "grievanceresistant," "3-6 rather than clinically indicated, for example, by ordering diagnostic tests or performing certain procedures, which are not strictly medically necessary. ${ }^{7,8}$ Whilst this claim is plausible and apparently accepted by the courts ${ }^{9-11}$ (cf. Hartshorne et al., ${ }^{12}$ p.522), there is limited empirical evidence in support of it so far as practitioners in the UK are concerned, $8,13,14$ although more extensive studies have been conducted in Canada ${ }^{15}$ and the United States. ${ }^{16,17}$ Yet this is not the only piece of legislation that uses reform of negligence law as a means of encouraging medical innovation. The "Right to Try" laws in the US including the Federal version, the Right to Try Act 2018 - effectively remove the patient's ability to sue in negligence over decisions to provide experimental

\footnotetext{
'University of Leicester, Leicester, UK

${ }^{2}$ University Hospitals of Leicester NHS Trust, Leicester, UK

${ }^{3}$ De Montfort University, Leicester, UK
}

\section{Corresponding author:}

Jose Miola, University of Leicester, University Road, Leicester LEI 7RH, UK.

Email: jose.miola@le.ac.uk 
treatment (although the legislation does not prevent the patient bringing an action if the treatment was provided in a negligent manner).

Our research seeks to address the gap in empirical knowledge by asking health professionals what they themselves see as the barriers to innovating responsibly. We sought to assess whether fear of the law was indeed the primary factor, but also what other obstacles might exist. Barriers need to be identified if these are to be overcome.

\section{Research method}

Focus groups were used to explore perceptions about barriers to responsible innovation. This is a time efficient method of generating data that cannot be obtained easily using techniques such as surveys. ${ }^{18}$ They are known to provide an "insiders" view of the realities of a topic and permit in-depth exploration of central issues whereby participants present their ideas, hear from others and then question one another about their insights. ${ }^{19}$

A purposive approach to recruitment was used and participants were invited by the research team. Five focus groups were held with a total of 26 participants. The groups were divided into specialisms: (i) surgeons (including those in orthopaedics, vascular, ear nose and throat, plastic and gastro-intestinal surgery); (ii) general practitioners (including specialist interest and private practitioners); (iii) allied health professionals (including a nurse consultant, matron, accident and emergency, outpatients and ward sisters); (iv) oncologists (NHS, researchers and private practitioners); (v) a mixed group (including rheumatologists, consultant physicians, anaesthetists and paediatricians).

The focus groups followed the model advocated by Krueger $^{20}$ in that all interviews commenced with an opening question on definitions followed by prompts and ending with a final specific question. The groups commenced by ascertaining participants' understanding of "responsible innovation." This was an important prequel in that some groups understood "innovation" as service improvement, while others saw it as technological or pharmaceutical developments. The closing question asked each participant to identify the key barrier to innovation from their own perspective.

Focus groups took place in vacant hospital offices using voice recorders and notebooks. All groups were facilitated by an experienced moderator and comoderator.

\section{Analysis}

Verbatim transcripts were generated within a week of each session and verified for accuracy to ensure that speech extractions could be mapped to follow individual contributions. Unabridged transcription-based analysis was chosen as the most rigorous strategy to adopt. ${ }^{21}$ Audio records were supplemented by field notes that were used to capture overarching themes that emerged during conversations.

The data sets were analysed using a variant of content analysis. Words and phrases were assigned to conceptual categories which revealed the significance of ideas by the frequency with which they pervaded discussions. $^{22}$ The data were categorised systematically according to initial themes that emerged. The transcripts from each group were analysed independently by two researchers to limit the potential for personal bias influencing the results.

The results and discussion are presented using overarching themes and illustrative quotations. The groups are indicated as follows: $\mathrm{S}=$ surgeons; $\mathrm{ONC}=$ oncologists; $\mathrm{MG}=$ mixed group; $\mathrm{GP}=$ general practitioners and $\mathrm{AHP}=$ allied health professionals. The numbers refer to specific participants in each group. The themes that emerged were (a) definitional issues, (b) fear of the law, (c) fear of the regulator, (d) access, (e) governance, (f) costs and (g) miscellaneous issues.

\section{Results}

Definitional issues. The groups defined innovation in different ways. Surgeons saw innovation was an intrinsic part of practical surgery. All had innovated in accordance with clinical need. They defined innovation widely, including doing things slightly differently to achieve the same result. Thus:

we can seal a vessel with clips, with sutures, with diathermy or you can use ultrasonics or advanced bipolar, so those energy devices aren't new but you are still achieving the same... So you are innovating in the technology that you are using but the end result would be the same. [S4]

Equally, oncologists saw innovation as part and parcel of all aspects of their work. Indeed, "[a] mantra we've got sort of out there on the wall is today's research is tomorrow's medicine" [ONC 1]. As one of them explained, "local trial recruitment is embedded within our service specification" [ONC 1]. Another stated: "our aspiration is that every patient, every entry into their journey will enter some form of research ... and whether at the diagnostic level or ... or treatment level or palliative care setting" [ONC 3]. Nevertheless, they did wonder whether they were exceptional, as they are a relatively new specialty and "probably one of the first that the expectation was that we would have a research degree" [ONC 2]. For these two specialties innovation 
was something to be actively sought, and was part of everyday clinical care.

In contrast, the other groups, particularly the GPs and AHPs, saw innovation as a reaction to problems with existing processes. GPs focussed on organisational issues, seeing innovation as increased efficiency and greater throughput of patients. It was more about "making the system work" [GP3]. Indeed, they saw themselves as in the middle of an inefficient system, and that innovation was necessary to enhance patient care. They felt pressured by lack of resources, and saw innovation as a way to mitigate this. The AHPs felt similarly that innovation was about improving patient care within the existing paradigm. One described innovation as "going that bit further with the patients and thinking of logistics and how you can meet their holistic needs, but you still need to work within the premises of policy and guidance." [AHP 2]. In other words, these groups felt constrained by external factors (for GPs it was resources and "the system," whereas it was policy and guidance for AHPs). They saw innovation as a means of improving patient care through efficiency initiatives. For these groups innovation was a necessity rather than an aspiration.

However, there was one common thread. As a paediatrician suggested: "innovation is the heart and soul of the working of any doctor, clinician worth his or her salt ... [b]ecause it amounts to problem solving" [MG 4]. How much it was sought depended on the size of the problem that needed to be solved.

Fear of the law. The law regulates the practice of all health professionals, and all the groups alluded to the manner in which the law influenced or affected their work. The extent to which the law was perceived as a barrier varied widely between groups. Fear of litigation was discussed, and GPs as a group were by far the most suspicious of the law and lawyers, being highly critical of what they regarded as bureaucracy and "tick-box" exercises they had to complete to avoid sanction. Some groups were concerned that other aspects of legal regulation, for example, patent rights negatively affecting clinicians' options to work with pharmaceutical companies. GPs were also concerned about coronial backlash in the event of patient death during innovative therapy. We consider the responses of each focus group below.

Mixed group: Intellectual property law as a potential barrier to innovation was identified by the mixed group. One participant expressed fear of losing control over intellectual property rights: “. . when you develop something, how do you trust somebody [not to] pinch your ideas, taking your ideas... intellectual property" [MG 2].

Another participant suggested that the law or legal requirements might deter pharmaceutical companies from collaborating with individuals with innovative ideas, for fear of financial liability either in the way of royalties, or in damages in event of being sued. One person stated: "I believe that the industry markets that makes devices or drugs ... often feel very, very suspicious because they don't want to encourage that [innovation] in case they become involved in the intellectual property of that idea."; and "[a pharmaceutical] company might say well we don't want you in on this because it's our drug and if we take you up on this, we might be liable and owe lots of money to you" [MG1].

Several participants indicated that fear of litigation had led them to act defensively, by extensively documenting advice and treatment given and consulting colleagues, or to act conservatively. One stated: "does it lead to defensive medicine...? ... If you look at the orthopaedic surgeons' letters, they are going to do a...hip replacement, and then there is a whole paragraph.... It cautions patients about infection, thrombosis, loosening, all of the above, it's all there, ok, but that's defensive medicine, we've been pushed into this position by litigation and by controlling authorities..." [MG1]. The effect of the legal profession was perceived as detrimental to medical practice: "There's one elephant in the room, ... this compunction to follow and compromise is born out of that hold that legal profession has had in medicine as of late and to that extent, I think we have forgotten that expense, our integrity..." [MG4].

Keeping good clinical notes and working in partnership with colleagues was seen as being beneficial, particularly if litigation ensued, as the courts were willing to recognise proper practice:

I think some of the litigation cases have gone the other way [that is in favour of the doctor], when the doctor has good notes and support and you know collaboration, working with colleagues and things like that, and in quite serious cases and the doctor has been shown to do everything they could in that situation, whereas before that would probably would have gone a different way with the pressures, so I think things are changing ... [MG3]

Ultimately, although some criticisms of the law were raised, none of the mixed group identified the law, or the fear of litigation, as being their principal barrier to innovation.

Surgeons: None of the surgeons specifically mentioned the law as the key barrier to innovation when asked to summarise their views. Increasing legal and regulatory oversight was, however, highlighted as having curbed surgical innovation in general:

...innovation, many can argue, was easier in the $60 \mathrm{~s}$ and $70 \mathrm{~s}$, where you could more or less do what you like, there wasn't laws and ethical committee, consent 
was not a big issue and you just say to the patients, this might help you, I'm prepared to give it a go, are you on board and they go or not.... The regulation round it is a fine line. It's got tighter and tighter, so you could argue that some of that is stifled innovation, because if you've got a new idea or whatever, ittakes a lot to get through all the regulations... [S5]

In relation to the fear of litigation, the surgeons indicated that they were far more concerned about the risk of being suspended by their employer, which would effectively end their career with catastrophic effects upon their livelihood: "I think for a lot of us it's not being sued, it's your livelihood, I suspect that you wouldn't be sued,... but your hospital may well suspend you for not going through the right [process committees]" [S5]; "Yes they would"[S3]; "That's the end of your career"'[S2];

I think that it's a livelihood thing. You would have to go and say to your wife, 'You have to go back out to work to pay for the mortgage, 'cause I'm unemployed... and the children have to go to Oxfam for shoes'... That's a big problem. [S5]

General practitioners: The GP group was by far the most trenchant in its criticism of the law and lawyers. They were particularly critical of the amount of form filling and documentation which they regarded as being required to prevent legal consequences but were an onerous check on their clinical discretion: " ... it's over documentation. And I think for medical legal purposes, the nurses have to show that they have been there, there were two nurses and different checks" [GP1];

... I think whoever makes this sort of law, I think they have to beresponsible....we are all tick boxing every day and we have to. Otherwise we get earmites.... it stifles [us] too much, so there needs to be some sort of responsible, sensible approach to individual freedoms really ... [GP5]

This group was the only one to mention fear of the coroner as negatively influencing their practice. One doctor expressed concern about coroners focusing upon whether a doctor had completed the Patient Questionnaire-9 (known as PWQ-9, ${ }^{23}$ an assessment tool designed to facilitate the recognition and diagnosis of depression in patients) when diagnosing potentially depressed patients, rather than upon the substance of the interaction between doctor and patient, and of the potential obloquy which might be heaped upon a doctor's head.

Fear of litigation, or of coronial intervention in relation to the end-of-life treatment of patients was also identified by the group as a barrier to innovation, with concerns that too many resources were directed at defensive over-treatment and monitoring of the dying:

I think that there is so much money being spent at the end of life when really if we went back to our roots, to following common sense, you know, keep patients comfortable...It is one of the blocks of innovation, I'm sure of it. [GP3]

"Lawyers again, they'll be on your back" [GP4]; "Check every fourteen days otherwise they will go to the coroners. ${ }^{24,25}$ That means aGP does a home visit every day..." [GP1].

One participant was particularly scathing of the perceived role of lawyers in inhibiting innovation, and suggested that the law's influence should be removed from medical practice:

I think the most dangerous people are lawyers. They have been a block to innovation, both in terms of litigation but also in terms of stopping more innovation in healthcare than we have... I think politics and law should be taken out of medicine, we should take control of medicine ourselves again ... [GP4]

Another expressed concern (based on personal experience) that the law was too patient-focused, which led to the reality of the situation and patient non-compliance being overlooked:

... medical law is very patient centred and protected.

So I had a patient who eventually asked for a claim because she was diagnosed with cancer and they felt she could have been diagnosed maybe twenty days earlier... But it did not see the fact thatshe delayed her hospital appointments, that she actually, she did not go a lot of times and did not take it seriously ... she did attend and then they discharged her and then they referred her again. They didn't see those aspects,... they think about those two weeks only. If you had followed the protocols, that's a two week referral, you're protected. If you had done an urgent referral, you're not protected...never mind the fact that it would have not have altered the course...common sense has completely gone... [GP1]

Allied health professionals: The views of the AHPs were markedly different from the other groups. Whilst restrictions imposed by professional regulation and policy guidance were discussed, there was no indication that the group regarded the law as a barrier to innovation. Apart from brief mention of the Charlie Gard case c6-29 $^{2}$ and the difficulty of balancing use of 
innovative treatments against the best interests of the child, the law was not specifically discussed.

Oncologists: Although Lord Saatchi suggested fear of being sued or prosecuted inhibited oncologists from providing innovative treatments, ${ }^{1}$ none of the oncologists expressed fear of litigation, or regarded the law as a barrier to its provision. Indeed, the group was generally critical of the Medical Innovation Bill, regarding it as unnecessary and unhelpful. One participant regarded the current regime as striking an appropriate balance between permitting innovation and protecting patients and practitioners:

I think that the way that we are regulated now, that if you do things properly, the whole point of having protocols, the whole point of going through ethics is so that you actually provide a safe place to innovate for both patients and staff. [ONC 3]

The only specific legal barrier to innovation mentioned by this group was in relation to clinical drugs trials. One member of the group indicated that increased European Union regulation had led to trials being moved outside of the United Kingdom: "We used to run a lot of studies here in the UK ... and then we had all the EU directives and that kind of thing and I would say that bureaucracy can be a problem ..." "[ONC3].

Fear of the regulator. A founding hypotheses of the project was that a potential barrier to responsible innovation might be fear of regulatory action from the professional regulatory bodies such as the General Medical Council (GMC) or the Nursing and Midwifery Council. The results suggest, however, that this was not considered to be a paramount barrier by participants.

Surgeons, for example, considered that practising within one's competence was a requirement of the GMC. Theoretically, this could therefore be a barrier to innovation. The paradox here, however, was that innovation, by definition (which from the surgical perspective involved mainly new devices and techniques), would be subject to a "learning curve" where competence could be established and aggregated gradually. Undertaking innovation could therefore be seen as the necessary aggregation of an evidence-base for a new product or device which would therefore fall outside the strict regulatory remit of the regulator, provided that the innovation fell within the ambit of good medical practice. Likewise, the oncologists perceived that the GMC requirements actually facilitated use of "off licence" products for the treatment of seriously ill patients.

These two specialist groups perceived innovation as a new way of treating the patient, rather than experimenting on patients. This reflects the view in Walker-Smith v $G M C^{30}$ where the Court acknowledged that the fundamental distinction between medical practice and research is based on the intention of the responsible health professional concerned. According to the court, where a

clinician departs in a significant way from standard or accepted practice entirely for the benefit of a particular individual patient, and with consent, the innovation need not constitute research, though it may be described as an experiment in the sense that it is novel and unvalidated. (Walker-Smith $v$ GMC, ${ }^{30}$ pp.11-12).

A contrasting view was evident from the GP, AHP and mixed groups where the majority felt that some of the primary barriers to innovation might have a secondary effect that could trigger regulatory action. Examples included the requirement to follow local policies and rules. Breach of these local requirements could lead to reporting to the regulator and prompt further action. Some participants stated that professional colleagues might refer an innovator to the GMC under the guise of "maverick" practice. A further barrier related to the over-burdensome and bureaucratic documentation that had become a routine part of practice. The time involved meant that there was little capacity for innovation which would encroach upon clinical time, thereby leading to mistakes which might result in regulatory action. It was felt that the primary role of the regulator would focus on practice rather than the detail of the innovation per se. There was an expectation that the detail around the innovation would be governed by regulatory functions carried outside the remit of the professional regulator.

Approximately half of the participants across all groups identified "employment regulation" as a significant barrier to innovation. This fear was premised on the growing corpus of local and employment rules within which individuals work. Factors such as tight managerial control and a hierarchical "top down" approach demanded rigid adherence to local policies that could act as a significant barrier to responsible innovation. This management ethos was considered to derive from a several factors, principally "efficiency of services" and "improvements [in delivery]." Breach of employment regulations could jeopardise the individual's career. In these circumstances, when there is already "enough to do," this could impose a further barrier to innovation which would add a further layer to the already burdensome structure of managerial control.

Access. An underlying barrier to responsible innovation was that of access, although this meant different things 
to different groups. For oncologists a shared concern was the ability to access continued (often unlicensed) treatment at the end of a clinical trial. Although suitable patients were often able to access treatment that was still under clinical development initially (whether on compassionate grounds or as part of a clinical trial) continued provision at the end of that period was more challenging. Reasons for this could be due to the length of time required for a drug to be approved. They could also be economic, particularly when the drug was expensive in terms of quality adjusted life years and had not been approved by the National Institute for Health and Care Excellence. It could also be due to lack of efficacy sufficient to approve the drug for general adoption. This is a fundamental aspect of many clinical trials. Although individual patients may experience benefits, a drug will not be adopted for general use unless its efficacy is proven sufficiently. Ultimately this could impact negatively on patient recruitment in that

[although] we've got a licenced drug, we know that it's amazing, but it is so expensive we can't access it and I do think that this is starting to have an impact on innovation, and people recognising that we've been involved in some clinical trials with some great drug that we can't use in our NHS practice.... and actually that's frustrating. [ONC 1]

Furthermore, even if a promising drug did receive early approval this was usually on condition that the manufacturer would continue to carry out registration trials to ensure that the drug was safe to use, "but they haven't always done those trials which has then been difficult" [ONC 2] which was mainly because of finance and limited potential markets.

For GPs, who were generally supportive of responsible innovation, the most significant constraints to access were time, space and money. Space was not limited to physical space but rather space to have ideas, and the ability to reflect upon what was needed for patients as well as service provision.

For surgical specialities, significant positive innovations tended to have resource implications. Initiatives such as robotic and keyhole surgery, for example, had high start-up costs and frequently led to centralised services. While this was useful for grant funding and clinical trials, this could affect access for patients who could not access centres of excellence.

Governance. A shared perceived barrier to responsible innovation was that of internal workplace governance systems. These were overly bureaucratic and timeconsuming procedures and governance mechanisms ostensibly designed for quality assurance purposes. All innovation, for example, irrespective of its simplicity or complexity required being "vetted" through tortuous processes: "...[it] still has to be approved before it can be actioned and I guess that's half the problem. If it sounds like a reasonable idea, give it a go" [MG5]; "... [you have] a zillion meetings" [MG3]; "[h]alf the time you think what's the point? It's going to take me 4 months, 20 meetings and actually all you're trying to do is save half an hour's work a week"[MG5].

More specifically, the existence of institutional research ethics protocols and requirements caused some participants to question whether the more ground breaking innovations of yesteryear could have happened in what they perceived to be today's more risk averse culture. "If you try to get ethics approval in this country, you struggle, you can't get it. Most people... do trials outside of the UK. And when they are done, they bring it back in the UK" [MG 3].

Although some national governance initiatives were considered to have potential to stymie responsible innovation ("we are expected to follow NICE guidelines and... if you don't follow NICE guidelines you have to justify why" [MG 3]), local policies and procedures were felt to exert a far greater impact. For one surgeon,

you've got [governance] at international and national and locals levels. For example, if you're going to introduce a new technique or technology into the Trust, you have to approach NIPAG [New Interventional Procedures Advisory Group]. [T] hat group will consider what's been proposed. [S3]

While the merits of robust governance was not criticised per se, the possibility of duplication of effort certainly was:

you have to go through a formal application process even if this is a procedure which has been accepted in places elsewhere... you have to go through that same process to allow approval and often of course, it will be on a case by case basis if they give you permission. And it's got be monitored very closely and audited. One can understand, of course, that it's with patient protection in mind but you've got to question whether, if there are plenty of other centres doing this, are we just reinventing the wheel each time. Shouldn't it be a centralised process? [S3]

In fact, some surgeons felt that there was a patient safety imperative for the introduction of more focused governance arrangements:

[We need] an audit or a registry... I am amazed that when there is a scandal [such as] breast implants, or hip 
re-surfacing, or whatever, [there is a call to] identify the patients who have had this and then there is this sort of, well, we don't keep records of that...nobody knows who's had what and I find that quite staggering. But despite all these regulatory checks, NIPAG Committees and all the rest of it, at the end of the day, when there is an individual problem,... we can't go on record and [see] who we need to recall. [S5]

There is "no simple way of saying: right, who across the country has had this?" [S3] Another shared concern of surgeons was that responsible innovation and clinical developments were less likely to emerge from European jurisdictions due to regulatory requirements and the need for early clinical data: "to get a European CE mark, you need clinical data ... you have to start somewhere" [S4]. This apparent "chicken and egg" situation was expected to push healthcare innovations to regions considered to be more supportive such as South America, China and Korea. Although oncologists agreed that biotech companies were more reluctant to innovate in the UK they felt that this was "because we are perceived to be very expensive" [ONC 3]

For general practitioners, existing governance regimes were perceived mainly as "top-down" control mechanisms that tended to fetter discretion rather than acting as a safety net for patients and staff.

Cost. All groups agreed on that cost was a significant barrier to innovation. This is unsurprising, but what is evident is that the notion of cost as a barrier is multifaceted. As noted by one surgeon, the cost of innovation had to be separated from the cost of clinical trials as money seemed to be available for the latter. But deep frustration was displayed towards both the government and hospital managers. According to one oncologist "[w]e do not have government funding for any level of innovation... we're always at the mercy of industry...[the]... mercy of biotech companies" [ONC 1].

Lack of money inevitably led to support for innovation being led by financial considerations rather than patient benefit. A mixed group participant (an oncologist) made this point explicitly:

How is innovation judged? How is it great? What's the detriment? $99 \%$ of the time it's financial. You want to say that you can do something and it's going to save loads of money. It's going to save us time, and time is money. [MG 3]

Another participant agreed, noting that the "usual mantra when any kind of originality was proposed was to propose a business case" [MG 1]. The surgeons, meanwhile, lamented that while there was money for some developments, such as new IT systems, in a "cash strapped NHS" this left little for surgical innovations such as robots.

Yet it was not just a general lack of money, or that money was being spent on wrong priorities. The theme that came through was lack of managerial foresight. According to several participants, it was not that enough that money would be saved by the innovation; instead the saving had to be made immediately. One participant expressed:

one of the big barriers is the short sightedness of the people making decisions. Because innovation may cost, but in the long run, yes, saves money. They don't care. They want to see at the end of this financial year, we have saved this.... And that's the problem. [MG3]

This was echoed by another who said:

Nobody thinks of anything for five years. So if you invest some money today, this year, and next year and next year, and the third year you save a lot of money, there's no appetite for that. Only appetite is for what you can see for this year... [GP7]

The problem of cost was not simply limited to how much money was allocated to innovation overall though this was certainly a factor. The problem also lies in the model of funding, where "quick fix" solutions are incentivised and longer term planning disincentivised. This is certainly a barrier against innovations that require initial outlay but that could produce tangible benefits later.

Miscellaneous issues. There were several other issues of note. An anaesthetist felt that one of the barriers to innovation was the difficulty in obtaining ethics approval for trials. Others, including an oncologist and several GPs cited a lack of time, particularly if developing innovations was not part of their primary role and therefore had to be carried out over and above their regular duties. Indeed, these two factors are linked. In both cases there was concern that before ethics committees and other decision-makers would consider proposals, some preliminary proof of concept was required. Unfortunately, compiling the evidence necessary to convince decision-makers to authorise innovation was prohibitive. Even more fundamental was that after completing a full patient list, there was simply no "brain space" left to think about innovation [GP5].

Another barrier recognised by several participants was the profession's innate conservatism that did not foster a culture of innovation. One oncologist disagreed that there was no time for innovation, but instead 
argued that there was no encouragement and therefore innovation tended to be reactive rather than proactive: "We always wait for things to go bad before we change" [MG 3]. This was echoed by another in that: "It's not like our Trust, not like there is a medical innovation fund,...there isn't that sort of encouragement" [MG 1]. But, ultimately, there was a feeling that even if individuals were not actively prevented from innovating, they would still be reluctant to do so. One AHP noted an inherent professional bias towards maintaining the status quo; although for one oncologist the problem was simple (though she was keen to exclude her immediate colleagues): "we're a bit conservative with a small 'c' about our approach to innovations... I just wonder sometimes whether people aren't risk takers" [ONC 1].

\section{Discussion}

The genesis of the project from which this paper derives was Lord Saatchi's Medical Innovation Bill, and in particular the claim that the only significant barrier to doctors providing innovative treatments was fear of the law. For that reason, we analysed the data in two parts: first, what doctors might fear in the form of the law and regulators; second, the influence of other factors. The results are instructive.

In relation to the law, we were surprised to find little support for the notion that fear of litigation was a barrier to innovation, and even less evidence that this was the principal impediment. What was even more unexpected was that the specialties who were least concerned by fear of the law were surgeons and oncologists, which were the groups who were uppermost in Lord Saatchi's mind when he composed his draft legislation. Even when fear of the law was mentioned as a potential barrier, as occurred in the mixed group, the "defensive practices" described, in the form of more careful note-taking and greater communication of risks to patients, were considered by the group to be examples of good practice that could protect doctors and patients. The notable exception was the group of GPs, who uniquely identified fear of the law as a potentially serious barrier to providing innovative treatment. However, even this finding has caveats. First, this focus group defined medical innovation in a way that was different to the other groups. For the GPs, innovation was less about treatment provision and more about processes and procedures to promote efficiency, such as greater patient throughput. Second, as primary practitioners and generalists, they are perhaps not the group that Lord Saatchi envisaged when he determined to loosen the shackles that he perceived to be were preventing innovation. Indeed, it is noteworthy that only one comment was based on personal experience, while other criticisms of the law appeared to be more generalised. From our findings, we suggest that for these participants, fear of the law was not a significant barrier to medical innovation, particularly for specialists.

The findings were similar in relation to fear of the regulators. Although not considered to be a primary concern, this could result from not following an obligatory rule or protocol. In sum, the barrier to innovation was not fear of regulatory sanction per se, but failure to follow mandatory rules or protocols. Again, it was notable that the surgeons and oncologists were the most relaxed about the role of the regulator, not least because they saw innovation as treatment rather than experimentation. This aligns with the view that where standard treatment will not work (as may be the case with advanced cancer), providing it would be futile and innovative treatment might be the only option. Neither the law nor the regulator would disapprove of attempts to innovate in such circumstances, and the specialists in our focus groups recognised this. ${ }^{31}$

If claims of fear being a barrier to innovation are unfounded, the other themes identified give a flavour of what the barriers actually are. The key point here is that these are interlinked. At the root of everything, in our view, is money. All groups identified cost as a key factor preventing innovation. This is unsurprising, but it does not make it any less true. It is clear that the NHS is currently experiencing a funding crisis, ${ }^{32}$ and it is neither unexpected nor unreasonable for managers to allocate funds to treatments that they know will work, rather than those that might. Moreover, this links with the idea that only innovations likely to save money immediately will be funded. Again, this speaks to a health service that is under pressure to save money today, rather than invest to save money tomorrow. This position is clearly antithetical to encouraging innovation. Equally, cost is a factor in relation to access to drugs and devices. Some participants explicitly linked the two, while others alluded to it by mentioning NICE, for example. As noted, we were unsurprised that cost was perceived as a key barrier, although the short-termism that it engenders is perhaps a less well-identified factor.

The other two barriers identified can be considered together: governance and a lack of culture of innovation. For the former, it appears that the logistical difficulty - in terms of the requisite approvals and paperwork, and the uncertainty of outcome, prove overly burdensome for many doctors. Essentially, they are reluctant to innovate when they see the length and difficulty of the journey to approval. This might lead to the lack of a culture of innovation that was referred to by participants. This factor was identified by the groups who felt constrained from innovating, but also the oncologists, who routinely innovate 
(although they were careful to exclude themselves from this cultural malaise). But if the journey to approval is perceived to be labyrinthine, it is not surprising that this culture is fostered. Lack of resources may again factor into these considerations, and might explain the difficulty in securing approval. But as far as the meetings and form filling are concerned, it is hard to argue for a loosening of the systems that are put in place (particularly in the case of research ethics, identified by one participant as a barrier to innovation) to protect patients.

\section{Conclusion}

We began this research with two intentions: to ask doctors what they felt the barriers to innovation were, and within that to focus on whether the claims made regarding fear of the law was indeed the principal concern as Lord Saatchi had claimed. As discussed, we did not find this to be the case, nor was fear of regulators. On the basis of our findings, we argue that there are two main barriers: lack of money and (perhaps consequently) the absence of a supportive culture. This is unsurprising and appears to link to other potential barriers such as managerial fiscal short-termism and an overarching culture of conservatism on account of the time and effort involved in making proposals that were unlikely to be accepted.

Yet, it is important to note that neither of the main proposals for legislative encouragement of medical innovation - the unsuccessful Medical Innovation Bill in the UK and "Right to Try" laws in the US - actually provide any money that is not already available. In that regard, neither would have made a difference to the participants in our focus groups. That said, Lord Saatchi has also spoken of his Medical Innovation Bill changing the culture of the medical profession to one that is more amenable to innovating. ${ }^{33}$ This is closer to what we found in our focus groups, but without more funding our research suggests that any attempt to change the culture would be fruitless.

We must acknowledge the limitations of our research. Our study was small, and we cannot claim that it necessarily definitely provides a representative sample of each specialty's views. We do feel, however, that they constitute an important snapshot of what the perceived barriers to innovation might be, and what they might not be, and that we can draw some tentative conclusions. What our research has done is identified what some of the barriers might be so that they can be assessed in more detail. We have also not considered any other issues, such as whether we want all doctors to seek to innovate, what constitutes responsible innovation (and conversely what constitutes irresponsible innovation) or whether removing some of the barriers would have the unintended consequence of allowing bad actors into the medical marketplace. These are important questions, and further research is required to answer them.

\section{Acknowledgements}

The authors gratefully acknowledge the support of the Wellcome Trust. They also wish to thank the focus group participants for their time.

\section{Declaration of conflicting interests}

The authors declared the following potential conflicts of interest with respect to the research, authorship, and/or publication of this article: AS has been in medical practice as a consultant for several years in the geographical area where this study was conducted; he has also held positions with national medical organisations that include the General Medical Council, National Clinical Assessment Service and NHS England.

\section{Funding}

The authors disclosed receipt of the following financial support for the research, authorship, and/or publication of this article: This research was generously supported by a grant from the Wellcome Trust (Grant number 205566/Z/16/Z).

\section{ORCID iD}

Tracey Elliott (D) https://orcid.org/0000-0001-9682-2284

\section{References and notes}

1. Saatchi Lord. Lord Saatchi Bill: we must liberate doctors to innovate. The Telegraph, January 26, medicalinnova tionbill.co.uk/lord-saatchi-bill-we-must-liberate-doctorsto-innovate/ (2014 accessed 29 April 2019).

2. Medical Innovation Bill 2013-14, Second Reading, Lord Saatchi, https://publications.parliament.uk/ pa/ld201415/ ldhansrd/text/140627-0001.htm\#14062743000565 (2014, accessed 29 April 2019). See Hoppe N and Miola J. Innovation in medicine through degeneration in law? A critical perspective on the medical innovation bill. Med Law Int 2014; 14: 266-273.

3. Tallis R. Hippocratic Oaths: Medicine and its Discontents. London: Atlantic Books, 2004, pp.253-254.

4. Tancredi LR and Barondess JA. The problem of defensive medicine. Science 1978; 200: 879-882.

5. McQuade JS. The medical malpractice crisis: reflections on the alleged causes and proposed cures: discussion paper. J R Soc Med 1991; 84: 408-411.

6. Merry A and Brookbanks W. Merry and McCall Smith's Errors, Medicine and the Law. 2nd ed. Cambridge: Cambridge University Press, 2017, pp.277-279.

7. Dingwall R and Fenn P. Is risk management necessary? Int J Risk Saf Med 1991; 2: 91-106.

8. Bertoli $\mathrm{P}$ and Grembi V. Medical malpractice: how legal liability affects medical decisions. In: $\mathrm{BH}$ Baltagi and $\mathrm{F}$ 
Moscone (eds) Health Econometrics: Contributions to Economic Analysis. Bingley: Emerald Publishing, 2018, Chapter 10; Available via University of York Working Papers WP 17/14: www.york.ac.uk/media/economics/ documents/hedg/workingpapers/1714.pdf (accessed 29 April 2019). The authors suggest that more sound empirical research is needed in this field.

9. Sidaway v Board of Governors of the Bethlem Royal Hospital and the Maudsley Hospital [1985] AC 871: Lord Scarman, 887 ("The danger of defensive medicine developing in this country clearly exists...).

10. Wilsher v. Essex AHA [1987] QB 730, Mustill LJ, 747 ("The risks which actions for professional negligence bring to the public as a whole, in the shape of an instinct on the part of a professional man to play for safety, are serious and are now well recognised").

11. ABC v St George's Healthcare NHS Trust [2017] EWCA Civ 336, Irwin LJ, [31] ("In my view, it is self-evident that there is a public interest in avoiding excessive litigation and in keeping to a minimum what one can call, in shorthand, defensive medicine...").

12. Hartshorne J, Smith N and Everton R. 'Caparo under fire': a study into the effects upon the fire service of liability in negligence. MLR 2000; 63: 502-522.

13. Ortashi O, Virdee J, Hussan R, et al. The practice of defensive medicine among hospital doctors in the United Kingdom. BMC Med Ethics 2013; 14: 42.

14. Summerton N. Positive and negative factors in defensive medicine: a questionnaire study of general practitioners. BMJ 1995; 310: 27-29.

15. Robert $\mathbf{J}$ and Pritchard S. Liability and Compensation in Health Care. Toronto: University of Toronto Press, 1990.

16. Kessler DP. Evaluating the medical malpractice system and options for reform. J Econ Perspect 2011; 25: 93-110.

17. Nahed BV, Babu MA, Smith TR, et al. Malpractice liability and defensive medicine: a national survey of neurosurgeons. PLoS One 2012; 7: e39237.

18. Duggleby W. What about focus group interaction data? Qual Health Res 2005; 15: 832-840.

19. Bloor M, Frankland J, Thomas M, et al. Focus Groups in Social Research. London: Sage, 2001, pp.5-6.
20. Krueger R. Focus Group Methods. Thousand Oaks, CA: Sage Publications, 2003.

21. Bloor M, Frankland J, Thomas M, et al. Focus Groups in Social Research. London: Sage, 2001.

22. Strauss A and Corbin J. Basics of Qualitative Research: Techniques and Procedures for Developing Grounded Theory. Thousand Oaks, CA: Sage Publications, 1998.

23. www.datadictionary.nhs.uk/data_dictionary/nhs_busi ness_definitions/p/patient_health_questionnaire-9_de. asp? shownav=1 (accessed 29 April 2019).

24. Regulation 41(1)(b), Registration of Births and Deaths Regulations 1987 (1987/2088) requires that the relevant registrar of births and deaths report a death to the coroner where it appears from a death certificate "that the deceased was not seen by the certifying medical practitioner either after death or within 14 days before death".

25. BMA. Confirmation and certification of death, www. bma.org.uk/advice/employment/gp-practices/service-pro vision/confirmation-and-certification-of-death (accessed 29 April 2019).

26. Great Ormond Street Hospital v Yates [2017] EWHC 972 (Fam).

27. Yates v Great Ormond Street Hospital for Children NHS Foundation Trust [2017] EWCA Civ 410.

28. Great Ormond Street Hospital for Children NHS Foundation Trust v Yates (No. 2) [2017] EWHC 1909 (Fam).

29. Gard v United Kingdom [2017] 65 EHRR SE9.

30. Walker-Smith v GMC [2012] EWHC 503 (Admin).

31. See in the legal context Simms v Simms [2003] 1 Fam 83; and in the ethical context Nuffield Council on Bioethics, Patient Access to Experimental Treatments 2018, http:// nuffieldbioethics.org/project/briefing-notes/experimentaltreatments.

32. Murray R. NHS finances on the brink. King's Fund, www.kingsfund.org.uk/blog/2017/02/nhs-finances-brink (2017, accessed 18 October 2019).

33. See: http://medicalinnovationbill.co.uk/ive-heard-lordsaatchi-and-others-talk-about-the-bill-changing-medicalculture-what-does-this-mean/ (accessed 29 April 2019). 